\title{
High-frequency brain activity: perception or active memory?
}

Tallon-Baudry and Bertrand 1 make a strong case for the significance of nonstimulus-locked high-frequency brain activity for cognition. When coherent figures are processed, large-scale neurophysiological recordings demonstrate stronger spectral power in the $20-60 \mathrm{~Hz}$ range compared with control conditions where no coherent objects are being recognized. Furthermore, their most recent study ${ }^{2}$ even indicates a role of highfrequency brain activity in short-term storage of coherent engrams, a view which can well be linked to data and theory about the role of memory networks in cortical active memory ${ }^{3}$.

With their investigations, TallonBaudry and Bertrand venture substantially beyond animal studies that focussed on cortical responses to simpler stimuli like the arrangements of bars slowly moving across the visual field. These 'moving-bar studies', and other research on high-frequency activity related to physically simple stimuli such as gratings, light flashes and tone pips, have provided important insights in cortical binding mechanisms. However, these studies are at the level of elementary perceptual processes. To test a theory about object representations and other meaningful cognitive entities, it is mandatory to explore the brain's responses to the corresponding stimuli: illusionary figures versus comparable incoherent arrays, physically very similar pictures which do versus do not evoke the perception of an object, and words whose shape and meaning have been learned versus meaningless but physically almost identical letter combinations for whom no previously learned representation exists. In demonstrating distinct modulation of high-frequency EEG and MEG responses to coherent and gestalt-like stimuli, Tallon-Baudry and Bertrand and their colleagues have made a major contribution to cognitive science.

We should, however, mention four issues raised in their article where, as to our view, data currently available call for modification and specification of their proposals.

\section{Feature binding versus associative} representations

Tallon-Baudry and Bertrand define their distinction between the binding hypothesis and a representational hypothesis in terms of bottom-up and top-down processes. Accordingly, topdown activation should apply to representations only. But one may well argue that top-down processes can also affect binding processes. It might be best to define the distinction in the following way: at the psychological level, 'feature binding' can be characterized as the linking of stimulus features according to cognitive principles ${ }^{4}$. The most important examples here are those proposed by Gestalt psychologists ${ }^{5}$, for example proximity, similarity, continuity (e.g. the property that two bars are aligned) and common fate (e.g. stimuli that move together). Evidence that high-frequency responses reflect such gestalt principles comes from numerous EEG studies, for example those showing that bars moving in the same direction elicit stronger high-frequency responses than bars moving in opposite directions ${ }^{4,6}$. Tallon-Baudry et al.'s finding of stronger high-frequency responses to Kanizsa's triangle compared with those to a non-triangle ${ }^{7}$ can also be related to feature binding, because only their Kanizsa triangle included aligned lines and thus satisfied the criterion of continuity.

In contrast to the feature binding view, one may posit that it is the individual's experience, not a general gestalt principle, which is crucial for high-frequency dynamics. Thus, highfrequency dynamics would be expected when learned associative representations are being activated. TallonBaudry and Bertrand's term 'object representation' is both too narrow and too wide: too narrow because stimuli other than objects, for example written and spoken words ${ }^{8-10}$, apparently elicit the same type of phenomenon. And too wide, because objects presented in an unusual way, for example faces presented not upright but horizontally ${ }^{11}$ can fail to elicit the high-frequency responses. Learned associative representations can be postulated for all entities that have been subject to learning These include many objects, TallonBaudry and Bertrand's dalmatian dog pictures, written and spoken words, and, more generally, perceptions and actions linked by associative learning. Notice that there are no Gestalt principles or other a priori cognitive principles that would predict feature binding for coherent items such as 'USA', but not for 'SAU'. Choice of the letter combinations that are meaningful in a particular language is arbitrary. However, the frequent occurrence of certain stimulus forms during language acquisition guarantees that cortical representations are being built up for them. Thus, a learned representation would 
become active if a stimulus fits the representation. An explanation based on associative learning also accounts for feature binding phenomena, because the coherent stimuli used in featurebinding-studies (e.g. bars moving together) are more common compared to stimuli used in the control conditions (bars moving in opposite directions).

In summary, we propose to reformulate the representational hypothesis as follows: activity of a neuronal representation formed by associative learning leads to induced high-frequency responses $>20 \mathrm{~Hz}$.

\section{Periodic oscillations versus complex} spatiotemporal patterns

The marked periodic oscillations that appear when animals are visually stimulated with coherently moving lines ${ }^{4}$ do not warrant that the same phenomena are actually picked up in EEG and MEG recordings during higher cognitive processing. Although Lutzenberger et $a .^{12}$ and Müller et al. ${ }^{13}$ demonstrated high-frequency dynamics in the human EEG similar to dynamics observed in the monkey, there is no conclusive evidence that these independent observations actually reflect the same phenomena. While dynamics in the highfrequency range of the EEG $(>20 \mathrm{~Hz})$ could be related to the modulation of rhythmic synchronizations, that is, coherent sine-like waves, it is equally well possible that they are caused by more complex spatiotemporal patterns of activity in large and distributed neuronal ensembles (see below). These spatiotemporal patterns can be periodic or aperiodic. There is certainly a range of electrophysiological phenomena that can underlie spectral dynamics.

Another important point is that stimuli activating representations of objects or words may produce physiological responses that differ in their nature from those induced by moving bars. The perceptual processes involving binding of elementary stimulus features and the activation of higher-level cognitive representations are not necessarily produced by the same brain mechanisms.

The influential neuropsychologist Donald Hebb ${ }^{14}$ suggested that cortical representations are strongly connected cell groups in which neuronal activity can reverberate in a well-ordered fashion. Synchrony of neuronal responses is but one special case of well-ordered activity. Evidence for non-oscillatory well-timed reverberatory activity comes from analyses of neuronal activity during cognitive tasks performed by monkeys ${ }^{15}$. Stimulus-specific precisely timed activity patterns have been observed, for example, during delayed matchingto-sample tasks, when the animal has to keep a particular engram active in short-term memory ${ }^{3,16}$. It may well be that neuronal activity rapidly reverberates in the many loops of an assembly whenever a coherent visual stimulus has been perceived and is 'kept in mind'. Such multiple reverberations are not necessarily periodic. Nevertheless, if they take place in a sufficiently large neuron population, they can cause fast changes of EEG or MEG activity. After filtering of the EEG/MEG signal, this change would emerge primarily as high frequencies dynamics. In essence, dynamics in high frequencies and changes of correlation coefficients obtained from large-scale recordings might be related to rapid complex spatiotemporal patterns rather than to oscillations in a narrow frequency band.

\section{Are high-frequency cortical responses} too late for perception?

There are early oscillatory phenomena reflecting elementary perceptual processes (latency $<100 \mathrm{~ms}$ after stimulus onset). These include stimulus-locked (evoked) activity in the auditory system closely related to the so-called middle-latency components of the evoked potential ${ }^{17}$, and oscillations in the retina and at later stages of the visual path that correspond to changes of physical stimulus features ${ }^{18}$. Furthermore, there are early high-frequency responses related to attention mechanisms ${ }^{19}$. Induced highfrequency activity, possibly reflecting the presence of learned associative representations activated by incoming stimuli, however, has been found at relatively late time intervals, usually $>250$ ms after onset of the stimuli ${ }^{20}$.

Tallon-Baudry and Bertrand state that induced high-frequency brain activity reflects perceptual and memory processes. We fully agree with the latter view but do not share the former. Stimulus-specific changes in evoked potentials and other physiological correlates of perceptual processes have been reported to occur early, significantly earlier than the corresponding differences seen in high-frequency responses. Differences in evoked potentials reflecting the distinction of physically similar but cognitively distinct stimuli (words versus nonwords; words of different types) occur as early as 100-200 ms post stimulus onset ${ }^{21,22}$. In contrast, differences in high-frequency responses to coherent words and incoherent pseudowords emerge around $300 \mathrm{~ms}$ (Ref. 8), almost exactly at the same time when Tallon-Baudry and Bertrand find spectral dynamics to $\mathrm{Co}-$ herent/incoherent figures ${ }^{7}$. The same point can be made for other kinds of stimuli as well. Electrophysiological processes corresponding to the access to cognitive representations occur before induced high-frequency dynamics. The initial access to a cortical representation might therefore be reflected in evoked potentials, whereas induced high-frequency responses might mirror processes related to active memory in reverberating cell assemblies following their stimulus-triggered initial activation.

Ruling out models of cortical arousal and attention

If a stimulus activates a piece of cortex, many things can happen there. A stimulus-specific representation might become active, but a more global increase of activity and of neuronal crosstalk is equally likely. Peter Milner ${ }^{23}$ proposed that sensory stimulation leads to non-specific activity increase in sensory cortices accompanied by oscillatory phenomena. At the cognitive level, this general activity increase could be related to an increase in arousal or attention to a particular modality, or even to one part of the visual field. A similar proposal has been made by Kirschfeld and colleagues ${ }^{24}$. Focussing of attention may correspond to an increase of the gain of a feedback regulation mechanism controlling cortical excitation. Such gain increase makes oscillations more likely ${ }^{24}$. Sheer had suggested earlier that focussed arousal is reflected in $40-\mathrm{Hz}$ EEG activity ${ }^{25}$. Results summarized in Tallon-Baudry and Bertrand's review ${ }^{1}$ can largely be explained by a focussed arousal or attention model. Enhanced high-frequency activity to Kanizsa's triangle ${ }^{7}$ was maximal at occipital sites, thus nicely fitting Milner's mode $^{23}$, while the more anterior maximum of high-frequency activity during the memory interval ${ }^{2}$ would not confirm Milner's view, but could be accounted for in Kirschfeldt's framework ${ }^{24}$ : areas more distant from the primary cortices might take more time to adjust their gains.

Is there empirical evidence that dynamics of high-frequency cortical activity actually relates to cognitive representations, rather than to arousal or attention? One possibility is to use stimuli whose cortical representations might be distributed over different cortical areas.

Consider visual stimuli that have been linked, by associative learning, to somatosensory perceptions in the left versus right hand. High-frequency dynamics and synchrony in visual and the respective somatosensory areas would be expected if these stimuli are being processed. Recently, Miltner et al. ${ }^{26}$ reported that increased coherence of EEG signals can reflect associative learning. After visual stimuli had been co-presented with somatosensory stimulation of the left or right hand, coherence increased between electrodes above visual areas and somatosensory areas contralateral to the hand stimulated. In this case, the associative links were reflected in a stronger correlation of high-frequency signals recorded over the cortical areas likely involved in association storage. This experimental outcome cannot be explained by an attention model, because correlation of activity at distant cortical sites were systematically related to learned contingencies.

In complementary studies on language processes, we used words reminding experimental subjects of actions or of visual stimuli ${ }^{27}$. Perception of these words, flashed on a computer screen, changed high-frequency EEG responses recorded over visual and motor areas. While over visual areas, 
stronger high-frequency activity was seen when visually-related words were processed, action words elicited stronger activity over motor and premotor areas. Similar topographic specificity was seen in evoked potentials, but these occurred before high-frequency dynamics. Semantic associations of the words were reflected in topographies of both evoked potentials and highfrequency responses.

In essence, physically similar stimuli that primarily differ in their learned associations elicited high-frequency dynamics and correlations at different recording sites, and likely in different cortical areas. These results can not be explained by a global attentional mechanism building upon stimulusevoked neuronal excitation. How should such an attention mechanism direct neuronal activity to different areas, based on cognitive properties of the stimuli? A theory of associative learning is necessary to explain that topographically specific high-frequency dynamics are a function of cognitive properties of incoming stimuli. Based on associative learning leading to the formation of distributed cortical-cell assemblies that include neurons in distant areas and produce reverberatory activity, it is possible to explain recent findings about induced high-frequency dynamics picked up in the EEG and MEG (Ref. 20).

Acknowledgement

Supported by the Deutsche

Forschungsgemeinschaft (DFG) and the Human

Frontier Science Program (HFSP).

Friedemann Pulvermüller, Andreas Keil and Thomas Elbert

F. Pulvermüller, A. Keil and T. Elbert are

at the Department of Psychology,

University of Konstanz, D-78457

Konstanz, Germany.

tel: +497531884242

fax: +497531882891

e-mail: friedemann.pulvermueller@

uni-konstanz.de

\section{References}

1 Tallon-Baudry, C. and Bertrand, O. (1999) Oscillatory gamma activity in humans and its role in object representationTrends Cognit. Sci. 3, 151-161

2 Tallon-Baudry, C. et al. (1998) Induced gamma-band activity during the delay of visual short-term memory tasks in humans J. Neurosci. $18,4244-4254$

3 Fuster, J.M. (1995) Memory in the Cerebral Cortex. An Empirical Approach to Neural Networks in the Human and Nonhuman Primate, MIT Press

4 Singer, W. and Gray, C.M. (1995) Visual feature integration and the temporal correlation hypothesis Annu. Rev. Neurosci. 18, 555-586

5 Kanizsa, G. (1976) Subjective contours Sci. Am. 235, 48-52

6 Eckhorn, R. et al. (1988) Coherent oscillations: a mechanism of feature linking in the visual cortex? Multiple electrode and correlation analysis in the cat Biol. Cybern. 60, 121-130
7 Tallon-Baudry, C. et al. (1996) Stimulus specificity of phase-locked and non-phaselocked $40 \mathrm{~Hz}$ visual responses in humans J. Neurosci. 16, 4240-4249

8 Lutzenberger, W. et al. (1994) Words and pseudowords elicit distinct patterns of $30-\mathrm{Hz}$ activity in humans Neurosci. Lett. 176, 115-118 9 Pulvermüller, F. et al. (1996) High-frequency cortical responses reflect lexical processing: an MEG study Electroenceph. Clin. Neurophysiol. 98, 76-85

10 Eulitz, C. et al. (1996) Oscillatory neuromagnetic activity induced by language and non-language stimuli Cogn. Brain Res. 4, 121-132

$11 \mathrm{Keil}, \mathrm{A}$. et al. Human gamma band activity and perception of a gestalt J. Neurosci. (in press)

12 Lutzenberger et al. (1995) Local 40-Hz activity in human cortex induced by visual stimulation Neurosci. Lett. 183, 39-42

13 Müller, M.M. et al.(1996) Visually induced gamma-band responses in human electroencephalographic activity: a link to animal studies Exp. Brain Res. 112, 96-102

$14 \mathrm{Hebb}$, D.O. (1949) The Organization of Behavior. A Neuropsychological Theory, John Wiley \& Sons

15 Abeles, M. et al. (1993) Spatiotemporal firing patterns in the frontal cortex of behaving monkeys J. Neurophysiol. 70, 1629-1638

16 Bodner, M. et al. (1997) Binary mapping of cortical spike trains in short-term memory J. Neurophysiol. 77, 2219-2222

17 Llinás, R.R. and Ribary, U. (1993) Coherent $40-\mathrm{Hz}$ oscillation characterizes dream state in humans Proc. Natl. Acad. Sci. U. S. A. 90 2078-2081

18 Neuenschwander, S. and Singer, W. (1996) Long-range synchronization of oscillatory light responses in the cat retina and lateral geniculate nucleus Nature 379, 728-732

19 Tiitinen, H. et al. (1993) Selective attention enhances the auditory $40-\mathrm{Hz}$ transient response in humans Nature 364, 59-60

20 Pulvermüller, F. et al. (1997) High-frequency brain activity: its possible role in attention, perception and language processing Prog. Neurobiol. 52, 427-445

21 Compton, P.E. et al. (1991) A cognitiveanatomical approach to attention in lexical access J. Cognit. Neurosci. 3, 304-312

22 Pulvermüller, F. (1999) Words in the brain's language Behav. Brain Sci. 22, 253-336

23 Milner, P.M. (1996) Neural representation: some old problems revisited J. Cognit. Neurosci. 8, 69-77

24 Kirschfeld, K. et al. (1996) Cortical oscillations and the origin of express saccades Proc. R. Soc. London Ser. B 263, 459-468

25 Sheer, D.E. (1976) The Neurophysiology of Learning Disorders (Knight, R.M. and Bakker, D.J., eds) University Park Press, Baltimore

26 Miltner, W.H.R. et al. (1999) Coherence and gamma-band EEG activity as a basis for associative learning Nature 397, 434-436

27 Pulvermüller, F. et al. Nouns and verbs in the intact brain: evidence from event-related potentials and high-frequency cortical responses Cereb. Cortex (in press) 